\title{
L'initiation rituelle javanaise et ses modes de transmission
}

Opposition entre javanisme et islam

Jean-Marc de Grave

\section{OpenEdition}

\section{Journals}

Édition électronique

URL : https://journals.openedition.org/tc/3032

DOI : $10.4000 /$ tc. 3032

ISSN : 1952-420X

\section{Éditeur}

Éditions de l'EHESS

Édition imprimée

Date de publication : 12 décembre 2007

Pagination : 85-124

ISSN : 0248-6016

Référence électronique

Jean-Marc de Grave, "L'initiation rituelle javanaise et ses modes de transmission », Techniques \& Culture [En ligne], 48-49 | 2007, mis en ligne le 20 juin 2010, consulté le 29 septembre 2022. URL http://journals.openedition.org/tc/3032 ; DOI : https://doi.org/10.4000/tc.3032

Ce document a été généré automatiquement le 29 septembre 2022.

Tous droits réservés 


\title{
L'initiation rituelle javanaise et ses modes de transmission
}

Opposition entre javanisme et islam

\author{
Jean-Marc de Grave
}

1 L'initiation rituelle javanaise, malgré l'intérêt socio-anthropologique marqué qu'elle présente, n'a jamais été étudiée auparavant (voir Grave 2001a: 7). John Pemberton (1994) ne parle pas explicitement de l'initiation martiale mais, prenant à contre-pied les monographies préexistantes depuis la période coloniale hollandaise, il montre comment celles-ci ont ignoré,

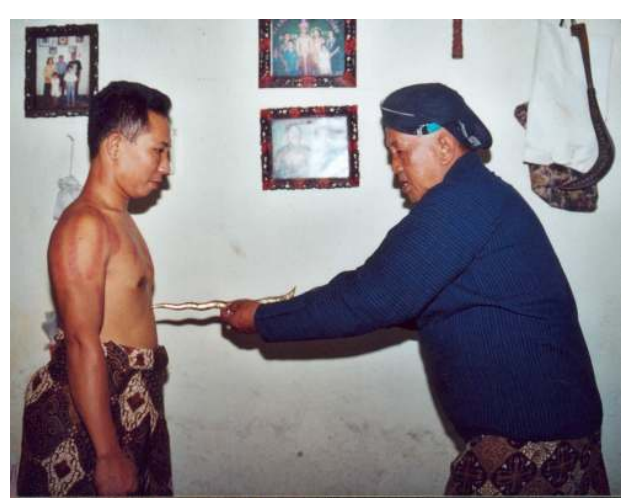
volontairement ou non, certains pans de l'activité rituelle javanaise: ceux qui évoquent l'activité guerrière endogène et certains de ceux qui expriment le caractère à la fois régénérant et sélectif de la nature. Pemberton récupère ainsi la dimension politique incluse, que le colonialisme, l'idéologie nationale indonésienne consécutive et l'idéologie des droits de l'homme -en tant que celle-ci s'élabore dans un environnement sociopolitique, sanitaire et militaire normalisé et comparativement surprotégé- avaient évincée des rituels comme pour désactiver un contenu ressenti menaçant ou déplacé.

2 Cet article a pour but de présenter les pratiques et les références d'un groupe d'initiation rituelle javanaise kanuragan et d'établir une comparaison avec celles d'une école de travail sur la" force vitale " (tenaga dalam) marquée par l'islam. Dans la partie consacrée aux valeurs sociales, j'introduis d'autres exemples d'écoles et de pratiques séculières, catégorisées kanuragan elles aussi, afin de renforcer la comparaison.

L'ensemble de ces écoles concerne une part importante des 240 millions d'habitants de l'Indonésie. Elles sont protégées par des personnages très influents liés aux domaines 
rituel, religieux, militaire, politique et économique, fréquemment pratiquants euxmêmes. L'aspect éducatif et formatif très valorisé de ces écoles et le rôle qu'elles jouent dans l'équilibre social sont inséparables de l'identité culturelle de la société javanoindonésienne. La formation dépasse largement le seul cadre d'un apprentissage de pratiques martiales, elle se présente comme un système de référence que l'on retrouve dans nombre de pays asiatiques. Outre l'activité cérémonielle, ce système concerne aussi les connaissances cosmologiques, les pratiques thérapeutiques, la religion, la philosophie religieuse et, partant, le rapport aux conceptions javanaises de l'autorité.

Il s'agit ici de tirer les conclusions qui s'imposent quant à la sélectivité des techniques employées et l'influence des valeurs respectives aux différents groupes en rapport à cette sélectivité : pourquoi tel groupe utilise-t-il telles techniques plutôt que d'autres ? Ainsi, au travers d'une activité, d'une pratique qui plonge aux racines socioculturelles javanaises, c'est aussi la configuration idéologique du système de valeurs d'une nation qui transparaît. In fine, l'article questionne le rapport dialectique qui s'établit entre pratique et représentation.

Le centre de kanuragan Trah Tedjokusuman : javanisme rituel ${ }^{1}$ Les trois niveaux de pratique

5 L'étymologie du terme kanuragan est certainement liée aux racines sanskrites raga et anuraga qui, dans leurs acceptions en javanais ancien mettent en avant les facultés émotionnelles du désir et de l'amour, alors que dans leurs acceptions en javanais moderne -suite à une évolution propre au contexte javanais-, elles mettent plutôt en avant le « corps physique » (raga), la domination et la force (cf. Grave 2001 : 18-19).

6 L'initiation rituelle kanuragan présente en son fondement deux éléments qui la caractérisent. Le premier est plutôt endogène à la personne et ses constituants. Il est lié aux cycles de vie et plus particulièrement à la naissance. Selon les conceptions javanaises, l'être humain naît avec quatre esprits germains qui l'accompagneront toute son existence (Grave 2001a : chap. III) et plus spécialement un "frère aîné » (kakang) issu de l'«amnios » (kawah) et un «frère cadet » (adhik), encore plus valorisé, issu du « placenta » (ari-ari). La pratique vise à utiliser la force de ces quatre esprits germains.

7 Le second élément - plutôt exogène-consiste en l'acquisition d'aji, lesquels désignent des connaissances qui sont aussi des capacités. À un aji particulier, comme nous allons le voir, s'attache(nt) une ou des caractéristique(s) spécifique(s). Le niveau de base de la pratique s'appelle kaenoman, composé javanais affixé depuis la base enom, «jeune ». Il consiste notamment à acquérir des formes d'invulnérabilité ; à ce niveau existe aussi une forme complémentaire qui a pour objet de se faire investir par un esprit d'animal. $\mathrm{Au}$ niveau intermédiaire, il s'agit de lier des contacts amoureux ou de protéger son environnement proche (foyer, travail...). Au niveau supérieur, le niveau kasepuhan -de la base sepuh, «âgé, sage, expérimenté »-, il s'agit de venir en aide aux personnes qui ont des problèmes, soigner les malades notamment, et de perfectionner les techniques de méditation pour augmenter son potentiel d'autorité spirituelle, de s'entraîner à la séparation du corps physique et du corps subtil (ngraga sukma), et de préparer son séjour post mortem dans le cadre des pratiques liées à la compréhension « de l'origine et du devenir de l'être » (sangkan paraning dumadi).

8 L'aji a une existence propre. Il se retransmet de personne à personne ou s'obtient en établissant une relation particulière avec le monde des esprits (alaming dhedhemit). Aji 
désigne aussi la formule spéciale -le mantra $a^{2}$ par laquelle on acquiert ou on invoque un aji. Dans les deux cas, l'aji est censé être une émanation du monde divin.

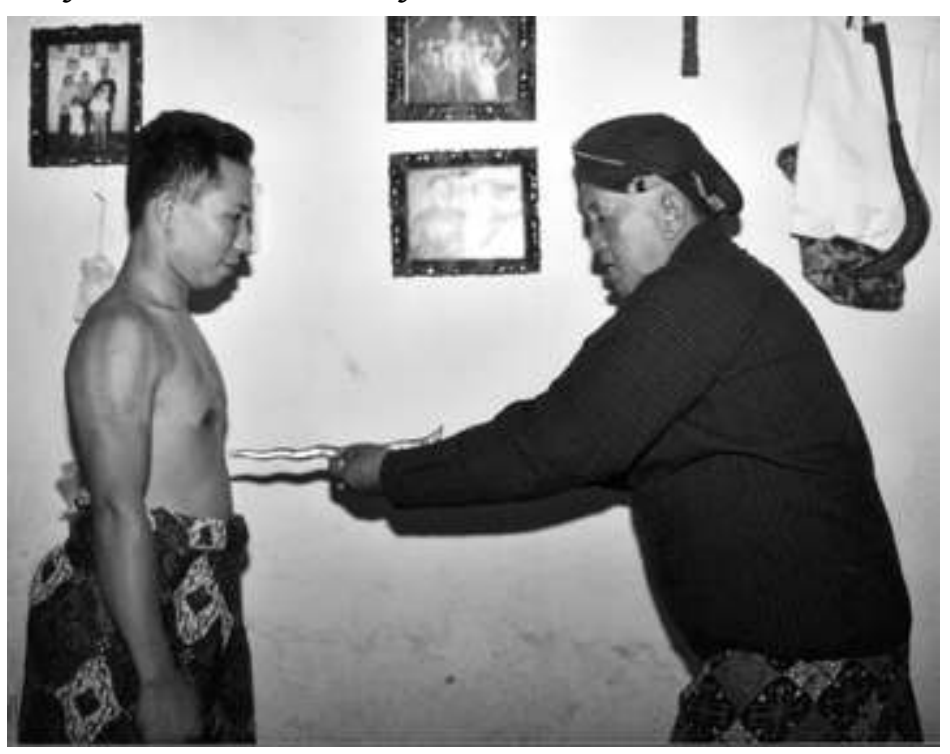

Figure 1. Mbah Budi se prépare à tester l'efficacité de l'aji Windu Kencana chez un élève.

Dans l'école Trah Tedjokusuman (TT) ici décrite, l'enseignement a lieu à proximité du palais royal de la ville de Yogyakarta, chez le maître appelé Mbah Budi.' Celui-ci prescrit à l'élève un type de jeûne spécifique à suivre pour l'acquisition d'un aji particulier. À l'issue de la phase de jeûne, lors d'un rituel d'initiation très élaboré, il baigne l'aspirant avec de l'eau florale consacrée, puis, après lui avoir fait répéter la formule de protection, teste son courage en lui assénant un coup de l'arme vis-à-vis de laquelle l'aji est censé rendre invulnérable. Le maitre retransmet alors la formule de protection de cet aji à l'élève, lequel offre en retour un repas rituel -appelé rasulan ou bancakan - au maître, à la famille de celui-ci, et aux autres membres de niveau « âné ». Le rasulan (du terme d'origine arabe rasul, "prophète ») fait référence à une catégorie de cérémonies communautaires dont l'objet vise à établir une relation avec un ou plusieurs type(s) d'esprits protecteurs donné(s) (Grave 2001a : 88-93). Il est notamment tenu à l'occasion du rituel de "nettoyage de village » (bersih dhusun), effectué lors de la récolte du riz. Il consacre la hiérarchie qui s'établit entre les esprits attachés à la personne, à la maison, au village, au royaume, et le Dieu macrocosmique englobant plutôt lié à la nation. Il consacre aussi les rapports aînés/cadets, en particulier le rapport parents/enfants, lequel exprime la relation qui s'élabore entre le maitre de TT (et son épouse, en quelque façon) et ses élèves. Les différents aji retransmis chez Trah Tedjokusuman sont reportés dans le tableau 1.

\begin{tabular}{|c|c|c|c|c|c|}
\hline $\begin{array}{l}\text { Forme d'aji } \\
\text { retransmise }\end{array}$ & $\mid \begin{array}{l}\text { windu } \\
\text { kencana aji } \\
\text { (« l'aji des } \\
\text { huit années } \\
\text { dorées ») }\end{array}$ & $\mid \begin{array}{ll}\text { aji } & \text { wali } \\
\text { sejati } & \\
\left(\begin{array}{ll}\text { l'aji } & \text { du } \\
\text { saint } & \\
\text { [musulman }] \\
\text { véritable }\end{array}\right)\end{array}$ & $\begin{array}{ll}\text { aji pangèlmunan } \\
\text { (der pang- } \\
\text { limunan: } \\
\text { «l'aji de } \\
\text { l'invisibilité») }\end{array}$ & $\mid \begin{array}{l}\text { aji keréwaja } \\
\text { (《l'aji du store } \\
\text { d'acier ») }\end{array}$ & $\mid$\begin{tabular}{||l|} 
ajian \\
klunthung \\
wesi \\
(« l'aji \\
l'enveloppe \\
d'acier $»)$
\end{tabular} \\
\hline
\end{tabular}




\begin{tabular}{|c|c|c|c|c|c|}
\hline Type de jeûne & $\begin{array}{l}7 \text { jours et } 7 \\
\text { nuits, } \\
\text { mutih : ni } \\
\text { sel ni sucre } \\
\text { ni viande }\end{array}$ & $\begin{array}{l}1 \text { jour et } 1 \text { nuit, } \\
\text { aucune } \\
\text { ingestion }\end{array}$ & $\begin{array}{l}2 \text { jours et } 2 \text { nuits, } \\
\text { aucune ingestion }\end{array}$ & $\begin{array}{l}3 \text { jours et } 3 \text { nuits, } \\
\text { aucune ingestion }\end{array}$ & $\begin{array}{l}\text { manger } 1 \text { seule } \\
\text { fois par jour à } \\
18 \mathrm{~h} \text { pdt } 3 \text { jours }\end{array}$ \\
\hline $\begin{array}{l}\text { Prière de base et } \\
\text { substance médiatrice }\end{array}$ & $\begin{array}{l}\text { Keblat } \\
\text { Papat } \\
\text { Kalima } \\
\text { Pancer } \\
\text {. encens et résine } \\
\text { de benjoin }\end{array}$ & 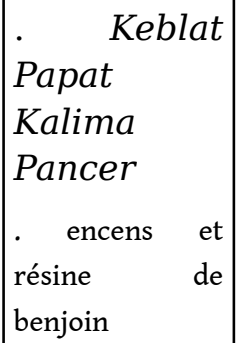 & $\begin{array}{l}\text { Keblat Papat } \\
\text { Kalima Pancer } \\
\text { - encens et résine de } \\
\text { benjoin }\end{array}$ & $\begin{array}{l}\text { Keblat Papat } \\
\text { Kalima Pancer } \\
\text {. encens et résine de } \\
\text { benjoin }\end{array}$ & $\begin{array}{l}\text { Keblat } \\
\text { Papat } \\
\text { Kalima } \\
\text { Pancer } \\
\begin{array}{l}\text { encens et } \\
\text { résine de } \\
\text { benjoin }\end{array}\end{array}$ \\
\hline Eau cérémonielle & eau florale ${ }^{4}$ & eau florale & eau florale & eau florale & eau florale \\
\hline Mantra spécifique & $\begin{array}{ll}\text { Sang } & \text { Windu } \\
\text { Kencana } & \end{array}$ & Wali Sejati & Pangèlmunan Sejati & Keréwaja & $\begin{array}{l}\text { Klunthung } \\
\text { Wesi }\end{array}$ \\
\hline Arme utilisée & $\begin{array}{l}\text { kriss } \quad \text { (arme } \\
\text { pointue) }\end{array}$ & masse d'arme & planche à clous & arme tranchante & $\begin{array}{l}\text { lance (arme } \\
\text { projetée) }\end{array}$ \\
\hline Repas cérémoniel & bancakan rasulan & $\begin{array}{l}\text { bancakan } \\
\text { rasulan }\end{array}$ & $\begin{array}{l}\text { bancakan } \\
\text { rasulan }\end{array}$ & $\begin{array}{l}\text { bancakan } \\
\text { rasulan }\end{array}$ & $\begin{array}{l}\text { bancakan } \\
\text { rasulan }\end{array}$ \\
\hline
\end{tabular}

Tableau 1. Les principaux éléments intervenant dans les pratiques de base

10 De façon générale, une période de jeûne doit débuter et s'achever après le coucher du soleil. Durant la période de jeûne, il faut éviter de se mettre en colère et il ne faut pas avoir de relation sexuelle. Il faut, en fait, éviter la dysharmonie émotionnelle. On doit donc s'efforcer de maintenir unis le sens de l'ouïe, le sens de la vue, le sens de l'odorat, le sens du goût et le « sens de l'essence vitale » (sukma rasa) auquel est lié le toucher et qui est au centre de la relation qui s'établit entre le monde divin et l'« essence vitale » (sukma) du pratiquant. Le jeûne n'est donc pas exclusivement alimentaire et l'état de concentration émotionnelle ainsi suscité est maintenu par le pratiquant lors de la cérémonie d'initiation. Les rituels sont tenus la nuit, de préférence les veilles de mardi ou de vendredi kliwon qui sont des nuits "sacrées" (kramat) se renouvelant chacune tous les trente-cinq jours ${ }^{s}$ et considérées comme propices à toute activité cérémonielle.

11 Le niveau intermédiaire est constitué de deux aji. La forme sri candra birawa vise -au travers d'un jeûne complet de vingt-quatre heures et d'une marche nocturne de douze heures accompagnée d'une méditation du maître- à consacrer les épousailles, dans la dimension suprasensible, du pratiquant et d'une jeune femme dont il a rêvé de telle façon qu'il en a eu une éjaculation. De cette fécondation médiatisée naît un « enfant nain [immatériel] » (bajang) qui protège le foyer du pratiquant.

12 La forme sri smaragama (" l'union amoureuse ») consiste, quant à elle, durant quarante jours, à prendre une douche nocturne après minuit et à ne pas avoir de relations sexuelles pour «neutraliser les attachements » et se rapprocher de la "perfection" 
(kasampurnan). Le smaragama se rattache à la fois aux pratiques génériques ngèlmu pangasihan qui visent à se faire aimer de personnes du sexe opposé et aux pratiques qui visent à affermir et développer sa position ou son rang; il marque l'entrée du pratiquant au «niveau des aînés » (tingkat kasepuhan).

Au niveau "aîné » sont retransmises les formes gineng (sur l'étymologie de gineng, cf. Grave 2001a : 74) qui consistent en "soins par la prière " (gineng panyuwunan) avec utilisation d'eau lustrale, mantra et rituels spécifiques; protection vis-à-vis des envoûtements ; initiation à la compréhension de «l'origine et du devenir "; protection contre les fauves et les animaux venimeux. La base de l'ensemble des prières utilisées pour le gineng est la prière Keblat papat kalima pancer, mais la prière Gineng du gineng panyuwunan devient elle-même une prière générique des pratiques de niveau kasepuhan. Le terme gineng fait référence à la poésie et au répertoire classique, où il s'agit d'un aji à la puissance quasi-universelle.

14 Les nuits de pleine lune les membres s'entraînent à «tirer le corps vital " (ngraga sukma), pratique évoquée aussi dans la littérature classique. Des formules et des positions spécifiques sont utilisées pour que le «corps vital » (sukma) se sépare du « corps substantiel » (raga).

15 Au niveau ultime, sont retransmises la forme wali sejati de niveau aîné, censée rendre le pratiquant invulnérable au feu; la forme sri gamana sari, liée à la connaissance préalable du gineng panyuwunan et se rattachant à l'« enseignement de perfection " (ngèlmu kasampurnan), vise à attirer le wahyu (terme javanais d'origine arabe: « révélation divine »), source d'autorité spirituelle dont la présence se caractérise par une lumière jaune au sommet du crâne; la forme nur cahya par laquelle s'établit la convergence des enseignements hindo-javanais et soufi au travers des notions respectives de Trimurti et d'unité de l'être. L'union de nur et cahya, selon Mbah Budi, forme le wahyu qui caractérise les rois, les héros mythiques et les grands mystiques; elle peut prendre la forme d'une étoile venant du ciel.

Le pèlerinage sur la tombe des maîtres du maître et des ancêtres fondateurs d'aji est une activité importante de l'école. Elle concerne principalement les membres de niveau supérieur et les sympathisants mécènes de TT. Ces visites s'ancrent dans la tradition javanaise globale du pèlerinage.

17 Notons encore que les pratiquants sont souvent, pour les niveaux de base, de jeunes Javanais âgés d'environ dix-neuf à vingt-cinq ou trente ans, qui viennent en groupes de villages proches de Yogyakarta. Les membres de niveau kasepuhan représentent une quarantaine de personnes âgées de trente-cinq à cinquante-cinq ans, voire plus pour les protecteurs de l'école. Outre les assistants du maître, le fils de Mbah Budi lui-même seconde fréquemment celui-ci. 


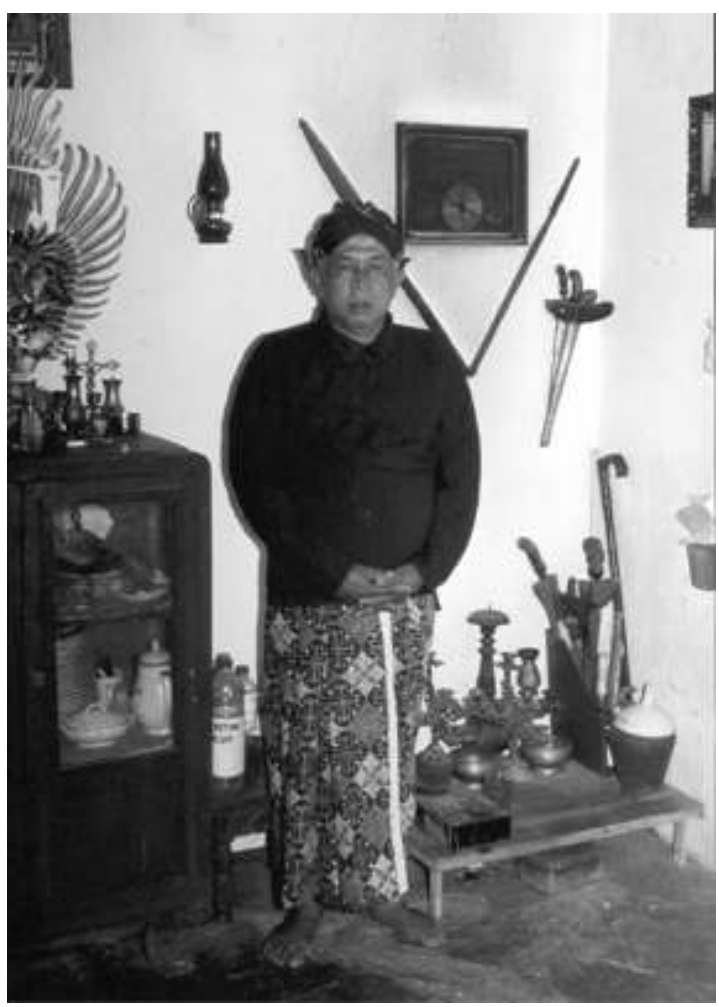

Figure 2. Mbah Budi dans la salle de prière, entouré de ses objets cérémoniels (brûloir à benjoin, kriss, pointes de lances, Garuda...).

Cérémonie du nouvel an javanais et activités extérieures du maitre

La nuit du nouvel an javanais est l'événement majeur de l'activité rituelle globale. Des déplacements considérables de population s'effectuent vers les lieux sacrés ${ }^{6}$ pour y prier, s'y recueillir, y faire des offrandes ou encore pour s'y promener en simple observateur. Trah Tedjokusuman effectue sa propre cérémonie pour l'occasion. En 1995, des costumes cérémoniels ont été loués pour les officiants (seize personnes) et on a fait venir de l'eau puisée sur divers lieux sacrés (des cinq puits du palais royal, notamment), soit dix pots en terre cuite de vingt litres chacun, dans lesquels on a adjoint les fleurs cérémonielles. Un repas cérémoniel à grande échelle a été préparé pour les visiteurs et pour les habitants du quartier. Des invités de marque -parmi lesquels des commerçants d'origine chinoise- venaient des villes de Jakarta, Surabaya et Semarang. Après les discours et les cérémoniels, la foule s'est dirigée vers les pots d'eau florale. Le maître a prononcé les prières et, aussitôt après, les jeunes gens du quartier, torse nu, se sont précipités sur l'eau pour s'en auto-asperger. Les autres personnes ont récolté de l'eau dans des récipients pour la ramener chez eux. Le rituel de « dispute » (ributan) -évoqué en introduction-s'est ainsi achevé et le repas a été servi.

19 Il faut souligner que le rituel de ributan est générique des cérémonies d'offrandes communautaires. Selon Pemberton (1994:18, 256-259), il fait référence à la lutte pour l'autorité et notamment celle qui concerne l'accession au statut de roi, ce qui peut être une des raisons pour laquelle il a été ignoré des monographies de la période coloniale et de la période suhartoïste. De plus, le kanuragan est, selon le Prince Joyokusumo frère de l'actuel sultan de Yogyakarta-, l'une des trois composantes traditionnelles qui président à l'accès au trône d'un des prétendants (entretien réalisé en 1995 à 
Yogyakarta). Les autres composantes sont les liens de parenté et l'implication dans les affaires du royaume. Le maître de TT est sensibilisé à cette tradition puisqu'il compte deux maîtres d'ascendance royale dont l'un était son « oncle aîné » (pakdhé) maternel, l'autre, Radèn Mas Harimurti, petit-fils du sultan Hamengkubuwono VII.

Quelque chose de la mise en place de l'ordre social se joue donc dans ce cérémonial qui s'élabore sous les portraits du président et du vice-président de la République d'Indonésie (Suharto et Try Sutrisno, en 1995), accrochés au mur où ils ne figurent pas d'ordinaire. Ces portraits surplombent la scène d'allumage des bougies et de l'encens par les invités de marque. Ils sont accompagnés des cinq images qui représentent la doctrine d'État du Pancasila et de l'emblème national du Garuda, l'aigle mythique du monde indien. Les chiffres 5 et 3 sont d'ailleurs omniprésents: chandeliers à 5 branches, eau des 5 puits, 5 principes du Pancasila; 3 grands bâtons d'encens qui dominent les chandeliers à 5 branches, soit la Trimurti javanaise et les 5 esprits germains de l'homme ; portrait du président Suharto, celui du vice-président et effigie du Garuda, lequel Garuda est représentatif de la totalité : les 5 symboles du Pancasila figurent sur sa poitrine et il tient dans ses serres la devise nationale Bhinneka Tunggal Ika, l'« Unité dans la diversité »; le 3 (les deux portraits et l'effigie) englobe ici aussi le 5 (le Pancasila) et relie celui-ci à l'ultime, au travers du Garuda divin, monture du dieu Vishnu. Ainsi, la classification traditionnelle javanaise telle qu'elle se retrouve dans la doctrine d'État, s'exprime ici au travers d'un cérémonial dont la dimension politique apparaît comme n'étant dissociée ni du rite, ni du mythe et, surtout, encadrée par un groupe dont l'activité - le kanuragan- est centrale pour la compréhension de l'Indonésie contemporaine.

21 Les rituels que le maître de TT maîtrise s'étendent aussi aux cérémonies liées aux cycles de vie, aux soins et à la protection à l'égard des esprits, ainsi qu'aux cérémonies ruwatan d'harmonisation cosmologique pour le mariage et la descendance (Grave 2001a: 35, 82). Dans la catégorie des rituels de protection figure aussi le «transfert d'objet sacré de famille » (nyedhot pusaka) de la dimension suprasensible à la dimension sensible, et inversement.

Ces dix dernières années, la notoriété du maître s'est notablement accrue. Ses consultants sont devenus plus nombreux. Des réseaux assez importants se sont développés avec les villes de Wonosari, Jakarta, Kediri, Semarang. On fait régulièrement appel à lui à l'occasion de la construction de complexes, de magasins ou de maisons, pour les protéger rituellement du vol, de la destruction, des catastrophes ou de la banqueroute. Il a aussi été contacté séparément par des représentants des principaux partis politiques' pour former certains de leurs membres aux techniques d'invulnérabilité.

Ces éléments ethnographiques serviront de base à la comparaison que je vais entreprendre à présent avec l'école Marga Luyu. Retenons de Trah Tedjokusuman son système relationnel de proximité de type familial, ainsi que son ancrage dans l'activité rituelle globale et dans le système de classification javanais.

L'école de tenaga dalam Marga Luyu'Présentation générale de l'école

La base du travail sur la «force vitale» (tenaga dalam) consiste en exercices respiratoires combinés à des mouvements de déplacement et des postures martiales de maintien et d'attaque. Le tenaga dalam est notamment pratiqué pour son effet bénéfique sur la santé et en vue de développer des capacités telle que la faculté de soigner certaines maladies. Ces pratiques sont probablement à rattacher au vaste ensemble 
d'« exercice du souffle » (pranayama, javanais ancien) d'origine indienne. Mes enquêtes sur le sujet indiquent que les postures utilisées seraient issues du pencak (art martial pieds-poings à percussion, très ritualisé et répandu sur l'île de Java), et encore plus probablement du pencak sundanais (Java Ouest), et se seraient stylisées dans la première moitié $\mathrm{du} \mathrm{XX}$ siècle en fonction du mode pratique propre au tenaga dalam.

Selon les témoignages des maîtres, jusque dans les années 1950 et 1960, la forme de transmission du tenaga dalam apparaît très ritualisée, comme dans l'exemple de Trah Tedjokusuman présenté ci-dessus. À partir des années 1960 et de plus en plus par la suite, jusqu'à aujourd'hui, les écoles adoptent le statut d'association et se développent fréquemment jusqu'à l'extérieur de leur zone culturelle d'origine pour couvrir parfois la majeure partie de l'Indonésie. Elles peuvent -à l'instar des écoles de pencak silat, l'art martial national indonésien- compter plusieurs milliers ou dizaines de milliers de membres et former jusqu'à plusieurs centaines de milliers de personnes sur plusieurs générations (cela concerne donc plusieurs millions de personnes sur une décennie). Dans certains hôpitaux d'État, on soigne les patients au tenaga dalam et un notaire, Bapak Daliso, a fondé en 1990 un mouvement fédératif des écoles dont le protecteur officiel est le sultan de Yogyakarta, Hamengkubuwono X, aujourd'hui gouverneur de l'Unité Territoriale Spécifique de Yogyakarta depuis 1999.

Concernant le rapport à l'islam de ces écoles, dans les années 1970 et 1980, elles étaient en majorité ouvertes aux seuls musulmans. Dans la deuxième moitié des années 1980 et, surtout, au cours des années 1990, un nombre important de nouvelles écoles ouvertes à tous se sont créées. L'influence de l'islam reste néanmoins globalement très forte sans que l'on puisse vraiment spécifier s'il s'agit d'une des tendances particulières de l'islam indonésien. La pratique du zikhr et d'autres techniques soufies semble néanmoins jouer un rôle important. Il n'est pas exclu qu'une partie de l'influence du soufisme se soit ainsi transposée dans ces écoles qui ont largement pénétré les milieux de l'islam progressiste.

L'école Marga Luyu (du sundanais marga : « chemin » et luyu : « noble ») passe pour être la plus ancienne école de tenaga dalam de Yogyakarta où elle est représentée par Bapak Effendi, grand propriétaire terrien et membre du conseil régional (DPRD). Outre l'île de Java, elle est aussi présente à Sumatera, à Célèbes et en Irian Jaya. Quoiqu'ouverte à tous, elle est fortement influencée par l'islam des confréries et le nationalisme soekarnoïste (c'est-à-dire lié au premier président d'Indonésie, Soekarno). Les créateurs des techniques sont présentés comme étant deux rois très importants des $\mathrm{XVI}^{\mathrm{e}}$ et XVII ${ }^{\mathrm{e}}$ siècles (Jaka Tingkir et Sultan Agung)'. La notion d'aji explicitée à propos de TT est évoquée chez Marga Luyu concernant la première forme apprise (voir plus bas) qui découlerait de l'aji Lembu sekilan, autrefois créée par Jaka Tingkir.

Description des techniques

Les techniques de base de Marga Luyu (ML) se présentent de la façon qui suit. L'inspiration est prise par le nez avant de commencer le mouvement. L'air est utilisé pour durcir la région du plexus solaire en combinant tension musculaire et rétention respiratoire (dans certains cas, l'air peut aussi être dirigé vers le ventre). Les débutants restent en apnée le temps d'effectuer quatre à six déplacements, les pratiquants confirmés effectuent dix à vingt déplacements, voire trente déplacements combinés. En fin de série, l'air contenu dans les poumons est intégralement expulsé, par le nez aussi. Dix mouvements spécifiques sont ainsi retransmis. À titre d'exemple, voici comment se présente le premier. 


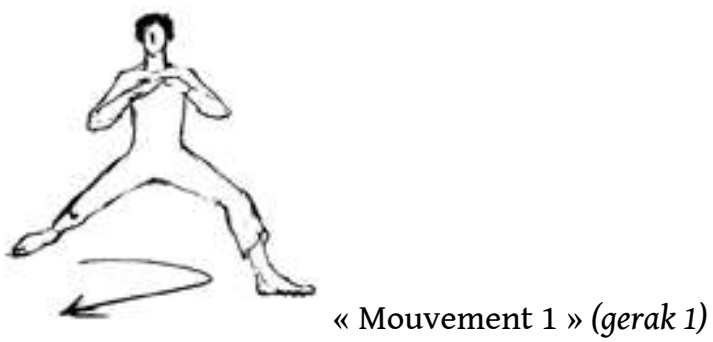

Figure 3. Le buste est droit, le regard fermement dirigé vers l'avant, mains levées, doigts serrés tournés vers l'avant. La position de campement est basse, pieds tournés vers l'extérieur

\section{(dessin Etsuko Migii)}

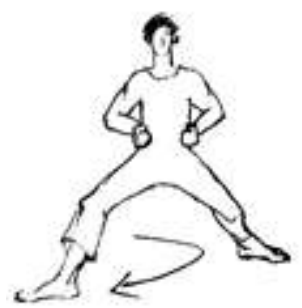

Figure 4. Le pied avance en arc de cercle et passe à proximité de l'autre pied. Les mains descendent contre les flancs bas. En restant contre les flancs, les mains pivotent sur elles-mêmes tout en se fermant en poings, paumes dirigées vers le haut, coudes des bras en arrière (dessin Etsuko Migii).

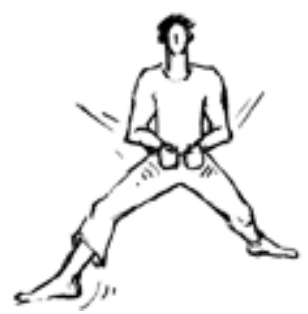

Figure 5. Le pied achève son mouvement en arc de cercle et se retrouve ainsi en avant.

Au moment où le pied droit arrive en fin de mouvement, le côté extérieur des poings émet un frottement contre l'abdomen d'un coup sec en diagonale vers le bas, de l'extérieur vers l'intérieur.

Lorsqu'ils se déplacent, les pieds restent en contact avec le sol. Ils émettent un frottement censé être productif de tenaga dalam

\section{(dessin Etsuko Migii).}

29 Certains mouvements semblent être inspirés de la prière musulmane (sholat) : 


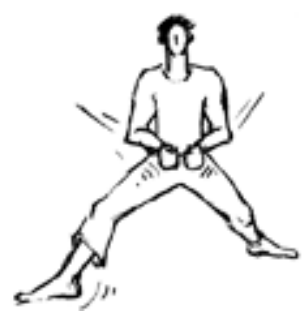

Figure 6. La position des bras et du buste au départ du mouvement 4 est similaire à la première position de sholat

(dessin Etsuko Migii).

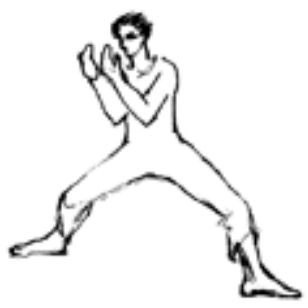

Figure 7. La position des bras et du buste au départ du mouvement 10 est similaire à la position d'invocation effectuée à l'issue de la prière

(dessin Etsuko Migii).

Lorsque les mouvements de base ont été effectués, il y a mise en pratique. Un des membres se met en position défensive tandis que les autres se ruent sur lui tour à tour en utilisant les jurus d'attaque (un jurus est une suite de mouvements offensifs et défensifs). Celui qui est en position défensive utilise des jurus défensifs, mais il reste campé sur place. Celui qui attaque doit être « en colère » (marah), point primordial pour que l'effet recherché soit obtenu. L'exécutant qui se défend prend une inspiration avant que la série d'attaques ne soit lancée. Il bloque l'air au niveau du plexus solaire, lequel est maintenu sous pression musculaire. Une partie de cet air est ainsi conservée en permanence pour maintenir la pression. Cette tension interne continue est censée produire l'élaboration d'une barrière immatérielle -autour du pratiquant- sur laquelle viennent butter les assaillants. Toutefois, si la colère des assaillants n'est pas réellement ressentie, la barrière est inefficace et l'attaquant entre en contact avec son adversaire, ce qui n'est pas le but recherché. Il est souvent difficile de susciter un tel état. Il s'agit à la fois d'une colère frénétique, mais malgré tout contenue et s'accompagnant d'une concentration extrême. Lorsque l'entraînement est terminé, la cérémonie de clôture est alors exécutée de façon similaire à celle d'ouverture.

Le tenaga dalam est fréquemment comparé à de l'électricité et nombreuses sont les écoles qui vont s'entraîner en bord de mer sur certaines plages, comme la plage sacrée de Parangkusumo où sont effectuées les cérémonies d'offrandes du palais royal à la Déesse des mers du Sud, Ratu Kidul. L'explication qui m'a été fournie concerne les vagues - très puissantes sur la côte sud de Java- qui, en s'enroulant et en s'abattant sur le sol, émettent de ce « courant électrique » aux alentours, lequel serait capté dans l'air grâce aux techniques utilisées. Un parallèle peut d'ailleurs être établi entre, d'un côté, le frottement recherché de l'air à l'inspiration et à l'expiration du pratiquant, le 
frottement de ses pieds sur le sol, et de l'autre côté le frottement des vagues sur le sable.

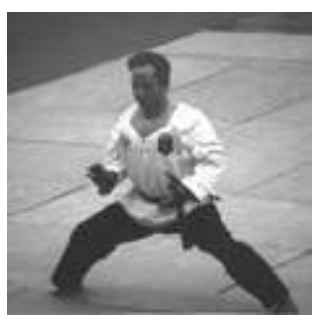

Figure 8. Forme respiratoire de type mouvement 3.

La fonction des dix mouvements retransmis se présente comme suit :

Mouvement 1 : Mouvement de maintien visant à accroître la résistance de base. Il peut être utilisé pour bloquer une action en cours : faire taire quelqu'un qui parle trop par exemple, ou calmer une personne en colère. Lorsqu'on met un mouvement en pratique au quotidien, on ne l'exécute pas, on applique la technique respiratoire adaptée au mouvement et, à un moment précis, on produit en image mentale une partie spécifique du mouvement (en général le dernier geste, lequel donne sens au mouvement). Par exemple, pour le mouvement 1 , on fixe des yeux l'objet, la ou les personnes visés. On inspire et on bloque l'air comme à l'entraînement. Pour donner le coup, alors que les muscles sont maintenus tendus, on effectue une poussée brusque de l'air comme pour expirer, mais on maintient les canaux respiratoires fermés afin que l'air rebondisse sur le plexus. À ce moment précis, on visualise le geste final du mouvement $1:$ les deux mains qui frottent vers le bas et latéralement la partie inférieure de l'abdomen, un geste de blocage. L'effet est en théorie simultané si le pratiquant maitrise la technique. Si ce n'est pas le cas, l'effet peut être faible, tardif ou nul.

Mouvement 2 : Il sert à soulever et à faire tomber, comme faire tomber quelqu'un en faisant passer l'« énergie » (tenaga) par le sol.

Mouvement 3 : Il sert à pousser, à projeter et à fermer. On peut utiliser ce mouvement pour « clôturer » un objet, c'est à dire le placer sous protection invisible.

Mouvement $4:$ Il a pour fonction d'ouvrir partiellement ou à échelle réduite (il fait paire avec le mouvement 9), ou de déchirer. Il peut être utilisé pour disperser (un groupe de gens par exemple).

Mouvement 5 : Il sert à tirer à soi quelque chose ou quelqu'un de visible, ne se situant pas à plus de cent mètres.

Mouvement 6 : Il a les mêmes effets que le 2 . On peut l'utiliser pour arrêter une voiture en marche ou quelqu'un qui court.

Mouvement 7 : Comme le 5, il sert à tirer quelque chose ou quelqu'un, mais situé à une longue distance. On peut l'utiliser pour contacter quelqu'un à distance par télépathie. Mouvement 8 : Il sert à détruire, à briser et à tuer. Utilisé de façon dosée, le mouvement 8 peut servir à soigner, ou bien rendre une personne soit amoureuse, soit bienveillante à l'égard du pratiquant.

Mouvement 9: Il sert à ouvrir intégralement et à déchirer, comme le 4 mais de façon plus puissante. Il peut être utilisé pour bloquer la circulation sanguine d'un adversaire. Mouvement 10 : Il sert à demander force, salut, joie et miséricorde au Seigneur. 


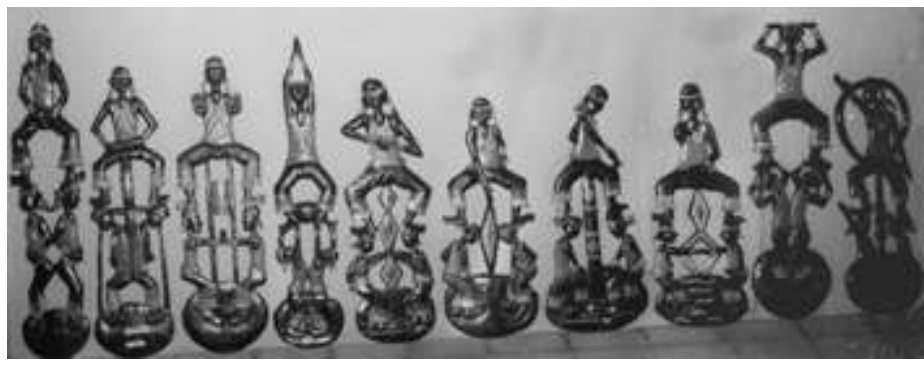

Figure 9. Figuration en bois peint des dix mouvements de Marga Luyu.

Structure des mouvements et techniques de soins

Nous obtenons donc deux séries complémentaires. La seconde série présente les mêmes caractéristiques que la première série mais intensifiées, élargies. Ainsi, si la première série est constitutive de la seconde, la seconde est néanmoins supérieure à la première. La hiérarchisation en valeur se présente donc ainsi :10, puis 1, puis 6 à 9 , puis 2 à 5 .

Ces deux séries s'établissent donc suivant un système à $4+1$ : pour la première série, ce sont les quatre mouvements $2,3,4,5$ plus 1 , pour la seconde série, ce sont les mouvements $6,7,8,9$ plus 10. Un lien structurel évident s'établit ainsi avec le système classificatoire javanais à base 4 évoqué à propos de l'école TT.

Les soins par la "force vitale» reposent sur la pratique des mouvements appris. Il convient seulement d'y adapter l'intention que l'on a de lutter contre la maladie. C'est l'«intention» (niat) mise en pratique qui permet de caractériser l'«énergie» nécessaire à la tâche à accomplir. Selon Bapak Effendi, l'« intention » liée à la " pensée créative » (cipta) s'exprime au niveau du « ressenti » (rasa) et c'est par celui-ci que l'on peut évaluer la nature du mal à combattre et en mesurer l'intensité. La maladie est, somme toute, considérée comme l'ennemi à supprimer. L'expression des intentions passe à la fois par le ressenti et la gestuelle attenante: synchronisation des mouvements, du regard, des membres (mains, tête, jambes) et des organes respiratoires.

Une phase de prière ouvre la séance. Ensuite, certains mouvements sont employés de façon combinée et répétitive, les gestes sont réduits, simplifiés et appliqués de façon rapprochée. Les mouvements les plus utilisés pour la guérison sont le 4 , le 3 , le 5 , le 8 , et le 10. Le mouvement 4 sert à " ouvrir " (membuka) l'endroit où siège la maladie. Le mouvement 3 sert à «déstabiliser" (mendorong) la maladie. Le mouvement 8 sert à "découper» (memotong) ou "déraciner» (mencabut) la maladie. Pour conclure, on effectue les mouvements 5 et 10 de façon alternée. Le 5 sert à «tirer» (tarik) la maladie, ou partie de la maladie, et le 10 à rendre à Dieu l'« énergie » néfaste que l'on vient de tirer. Pour « fermer » (menutup) ce que le mouvement 4 a ouvert en début de séance, on utilise le mouvement 3. Pour conclure la séance, une phase de prière est tenue afin de remercier le Seigneur. Le maître peut aussi utiliser des plantes. Il les mélange, en fonction des différents types de maladie, et leur insuffle son propre tenaga dalam afin d'intensifier les effets curatifs. ${ }^{10}$ 


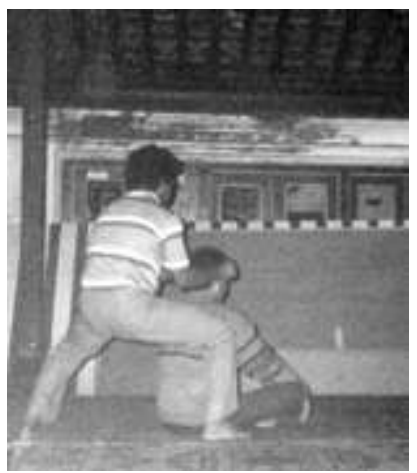

Figure 10. Soins de mal de tête par un membre de Marga Luyu (tension musculaire du soignant).

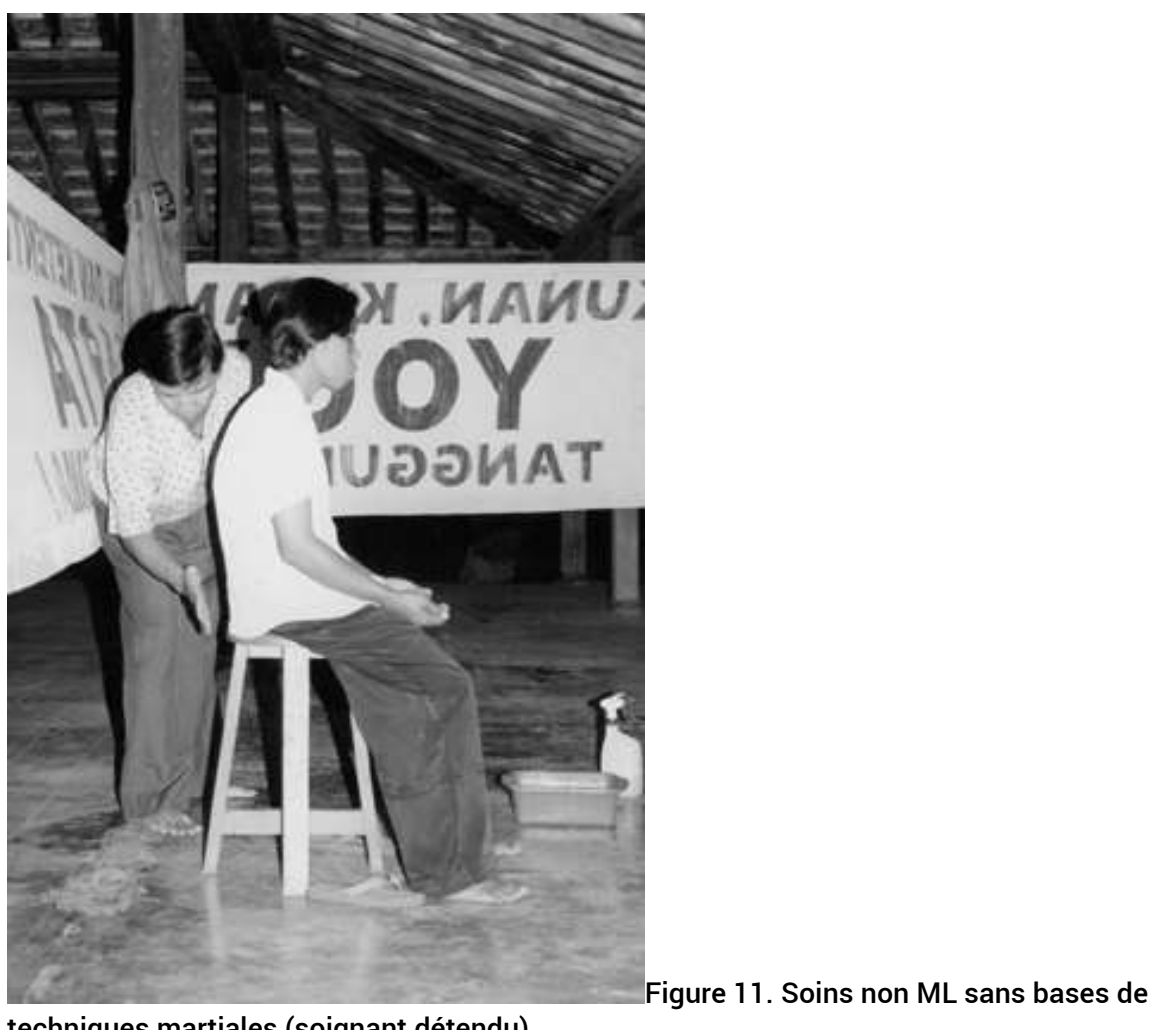

techniques martiales (soignant détendu).

Déroulement de l'entraînement, système de transmission

Un âge minimum de dix-sept ans est requis pour pratiquer. Il est admis que la manipulation du tenaga dalam peut s'avérer dangereuse pour la santé des enfants et des adolescents. Lorsque la série de 10 est maîtrisée, les élèves accèdent au niveau supérieur à l'issue d'une cérémonie spéciale dirigée par le maitre (voir plus bas). Les élèves qui ont suivi cette première cérémonie peuvent pratiquer les mouvements combinés et assister les entraîneurs.

Pour les niveaux suivants, la formation tourne autour de la transmission du jurus $R$ (lequel comporte vingt-deux mouvements de déplacement), qui peut prendre plusieurs années. La transmission de ce jurus se déroule en deux fois; elle se clôt par une cérémonie d'accession spécifique et est soumise à des conditions qui dépassent la simple activité de membre de l'école : pratique du jeûne et bénévolat dans le domaine de l'aide sociale, afin de développer le sentiment de «compassion» (prihatin), 
notamment. À l'issue de la seconde cérémonie d'accession du jurus $R$, les pratiquants deviennent des assistants du maître à part entière.

Les niveaux ultimes ont pour noms : tingkat pelebur et tingkat tarekat. Pour y accéder, les émotions doivent être parfaitement maîtrisées. Un entraîneur m'a expliqué que n'étant pas marié et n'ayant pas un métier stable, il refusait de lui-même d'accéder au niveau pelebur. En 1995, les membres de tingkat pelebur étaient au nombre de vingt-cinq pour la région de Java Centre et ceux de tingkat tarekat au nombre de cinq pour toute l'Indonésie (l'école revendiquait alors 500000 membres, d'après les données dont je dispose, elle devait en compter en tout cas plus de 100 000). Au niveau pelebur (du javanais lebur : "réduire, annihiler»), les pratiquants sont capables de lutter efficacement contre les "forces mauvaises" (kejahatan dan ilmu hitam), soit curativement, soit préventivement. Le niveau tarekat fait explicitement référence aux confréries soufies; les capacités en cause sont notamment celle d'ubiquité et celle de lire les pensées : l'« énergie » se déplace là où le pratiquant la sollicite par sa " volonté » (niat).

En 2001, l'école comptait encore très peu de professionnels : quatre en tout, dont le responsable général de la région de Célèbes Sud et le fils aîné du maître. Les personnes qui pensent qu'on ne doit pas gagner d'argent grâce à de telles pratiques sont en effet largement majoritaires à Java.

Activités de l'école

Les cérémonies d'«accession" (wisuda) qui ponctuent l'apprentissage des dix mouvements de base ont lieu chez le maitre (il se rend néanmoins sur place, si la région est éloignée). Elles regroupent parfois plusieurs centaines d'élèves et sont volontairement tenues à l'issue de la " prière » (sholat) des musulmans. Elles consistent en la transmission d'une "formule" (azimat) à utiliser en combinaison avec la respiration -de façon similaire à la pratique du zikhr soufi-, en l'ingestion d'un morceau de papier sur lequel est inscrite une formule secrète, en une harangue et un discours explicatif du maitre, et en un repas pris de concert.

51 Chaque année, l'ensemble des centres régionaux se réunit à Yogyakarta. Ces réunions sont organisées dans des stades et attirent parfois jusqu'à dix mille membres. On y effectue des entraînements à grande échelle et des démonstrations ; les repas sont pris en commun, des groupes d'élèves sont intronisés, des discours sont prononcés. Cette activité est à mettre en parallèle avec celle du nouvel an de Trah Tedjokusuman, la différence étant qu'ici, c'est l'importance de l'école qui est mise en avant.

52 Une autre activité importante, quoique plus intime, est le pèlerinage sur la tombe du sultan Agung, le plus important monarque de la dynastie Mataram, qui passe pour être le créateur des formes enseignées à l'école.

Comparaison des systèmes de transmission des écoles Trah Tedjokusuman et Marga Luyu

53 Les activités de l'école Marga Luyu se présentent comme étant rituellement moins denses que celles de Trah Tedjokusuman; sa structure de transmission est néanmoins très proche. Nous y retrouvons l'équivalent d'un niveau «jeune" et d'un niveau " aîné ", avec des catégories de pratiques similaires -martiales dans le premier niveau, soins et mystique dans le second. Dans les deux cas s'établit une référence au soufisme au niveau ultime de l'enseignement. Ce point est à mettre en relation avec la doctrine javanaise d'«Union du serviteur avec le maître », notamment liée au palais royal 
(suivant la doctrine soufie d'Unité de l'être, chaque être est une expression particulière du même Dieu, cf. Woodward 1989 ; Grave 2001 : 76-79).

En parallèle, nous trouvons les marques partiellement recouvertes du système de correspondance javanais à base 4 au travers de la classification des (deux fois $4+1=) 10$ mouvements de base (il est aussi présent dans les 4 couleurs du logo de l'école ML blanc, rouge, noir, jaune- et les caractéristiques qui les accompagnent -pureté, courage, puissance, noblesse). Ces éléments à $4+1$ termes sont en relation avec des éléments groupés de façon tripartite. Par exemple, les deux séries de $4+1$ mouvements sont en relation avec les 3 caractéristiques respiratoires "inspiration, rétention, expiration ». C'est par la rétention respiratoire que l'imprégnation du tenaga dalam opère ; or le tenaga dalam - «force vitale » unifiante- habite tout être vivant et implique de ce fait une relation particulière à Dieu et au cosmos. De cette façon, le rapport du 5 au 3 avec le 5 orienté horizontalement (pratique des mouvements) et le 3 orienté verticalement (lien au cosmique) transparaît comme chez TT où on la trouve dans le culte rendu par Ego aux 4 esprits germains ( $4+1$ éléments), lequel Ego est encore en relation de germanité avec son "frère aîné le ressenti " (kakang sukma rasa, lié à l'amnios) et son «frère cadet le corps vital profond" (adhik sukma sejati, lié au placenta), soit 2+1 éléments.

55 La relation « intérieur-extérieur » que l'on trouve avec les aji chez TT est aussi présente chez ML au travers du travail sur la respiration (j'y reviendrai). La notion même d'aji est évoquée chez ML à propos de l'héritage du monarque javanais créateur de ce qui est devenu le mouvement $n^{\circ} 1$.

Ce schéma lié à l'activité rituelle javanaise transparaît donc chez ML même si les éléments constitutifs javanais ne sont pas formellement mis en avant. Il reste que certains éléments structurants apparaissent fortement atténués. C'est notamment le cas de la relation "homme-femme ", très marquée chez TT au niveau intermédiaire, présente mais à peine évoquée chez $\mathrm{ML}$, ce que l'on peut a priori mettre en rapport avec le recouvrement idéologique dû à l'islam et à la modernité, avec le fait attenant que les femmes sont ici admises à la pratique (au contraire de chez TT) et représentent environ $30 \%$ des effectifs. L'école ML présente ainsi une riche source d'informations quant à la conformation de la société javanaise telle que celle-ci s'ancre dans le développement national indonésien.

Pour détailler la comparaison, je distinguerai trois éléments intervenant dans le processus. Dans un premier temps, je parlerai plutôt du point de vue des pratiquants et dans un second temps, plutôt du point de vue du ou des transmetteur(s). Il s'agira ensuite de mettre en perspective l'élément technique dans sa dimension sociale élargie. Les techniques de base

58 Nous avons pu constater que chez TT, la technique de base est celle du jeûne alimentaire (comme point d'appui du jeûne total), tandis que chez ML, le travail de base est celui de respiration en mouvement. Il faut néanmoins préciser que dans les deux cas, il s'agit bien d'aiguiser son potentiel de concentration.

Chez TT, la pratique est périodique. Un élève peut difficilement entreprendre d'effectuer par an plus de six ou huit jeûnes visant l'acquisition d'aji. Chez ML, en revanche, la fréquence de pratique est bi-hebdomadaire. On constate donc que le rapport à l'ingestion alimentaire exprime une temporalité de pratique différente de celle qui concerne l'acte respiratoire. 
Chez TT, parmi les éléments complémentaires utilisés - ceux qui renforcent le jeûne de base-, il y a tout d'abord les formules (aji ou mantra). On les retrouve chez ML mais pas de façon aussi élaborée ; au niveau de base, il n'y en a qu'une très brève (quelques mots d'arabe), tandis qu'au niveau équivalent chez TT, il y en a au moins huit, dont certaines sont relativement longues.

61

$$
\text { des lieux sacrés. Nous retrouvons ceux-ci chez ML, mais aux niveaux de pratique élevés, }
$$
et même alors, on parle toujours plutôt de prière que de méditation. Les éléments complémentaires à la pratique de base du tenaga dalam sont liés à la qualité de l'environnement «naturel» (alami) et de l'air "pur» (murni). Il est vrai que cette qualité recoupe parfois des phases d'entraînement sur des lieux sacrés, comme la plage de Parangkusumo, mais il ne s'agit pas dans ce cas d'un pèlerinage véritablement rituel. Nous pouvons comparer ces éléments à l'eau florale utilisée chez TT en ce qu'ils sont constitutifs du lieu, même s'ils semblent culturellement moins marqués.

De façon plus globale, les questions de maîtrise respiratoire et alimentaire se rattachent toutes deux au rapport qui s'établit entre l'intériorité et l'extériorité du pratiquant : il s'agit de contrôler et de maîtriser les éléments qui pénètrent le corps. Cette maîtrise est liée à la conception javanaise d' "équilibre » (timbang). Il y a l'équilibre du chaud et du froid, lequel détermine la classification des aliments en relation avec la température extérieure, le climat et les humeurs corporelles (Friedberg 1985 ; Jordaan 1985). Les facultés humaines, la pensée créative (cipta), le ressenti (rasa) et la volonté (karsa), doivent être, idéalement, maintenus en état d'équilibre. On remarque que, même aujourd'hui, le «corps » (raga) n'est pas associé à ces facultés. La première raison est que dans les conceptions javanaises classiques - lesquelles perdurent en partie de façon explicite et de façon très marquée au niveau empirique-, le corps physique se présente comme une émanation des facultés subtiles, même s'il représente par effet retour une base de travail pour affiner celles-ci (au sujet des différentes notions cognitives javanaises, cf. Grave 2001 : 126-128). La seconde raison, attenante à la première, est que le corps n'est jamais pensé comme une entité autonome: il n'est dissocié ni des relations sociales, ni des facultés évoquées ci-dessus (cipta, rasa, karsa). L'introduction d'une opposition duelle " corps-âme » (lahir-batin) est due à l'islam, mais au niveau du vécu quotidien, elle reste très théorique. L'équilibre corporel est d'ailleurs valorisé, au point d'être au centre des systèmes de formation traditionnels (arts martiaux, théâtre, danse, tenaga dalam... cf. Grave 1996a/b, 2001a/b, 2003).

Par rapport à cela, notons que l'éducation traditionnelle - soutenue par les relations au quotidien- vise à ne pas ressentir, penser ou vouloir n'importe quoi n'importe comment. Les pensées, par exemple, sont considérées comme évoluant à une "fréquence » (getaran) proche de celle des « créatures subtiles » (badan alus), maîtriser ses pensées, c'est donc aussi éloigner les mauvaises influences de soi. Le travail sur la concentration, que l'on trouve dans les deux écoles, s'inscrit dans cette perspective javanaise. Des références explicites sont même parfois utilisées chez des entraîneurs ou des hauts responsables de ML.

64 Ainsi, les éléments relevés ci-dessus -le rapport au temps et à l'environnement, notamment- indiquent chez TT un ancrage direct dans le temps mythique (cf. supra). Comparativement, la pratique et les techniques s'appuient chez ML sur des éléments plus neutres. Par son insertion marquée aux calendriers national et musulman laquelle est rendue possible par le type de techniques utilisées-, la relation au temps y 
est plus condensée car détachée des autres activités et donc détachée d'une perception globale du temps. Je développerai ce point dans le paragraphe consacré aux valeurs sociales.

La transmissionLa relation maître-élève et les conceptions javanaises de l'apprentissage Le maitre de TT sélectionne les apprentis initiés de façon assez rigoureuse. Disons qu'il se situe à mi-chemin entre une forme sélective de type traditionnel et la nécessité de maintenir vivants savoirs et savoir-faire «javanistes" (kejawèn). Le premier type de sélection consiste à trier les élèves sur le volet. Il n'y a encore pas si longtemps, l'apprenti résidait chez le maître, d'où la nécessité pour ce dernier d'évaluer le caractère éthique et la motivation profonde de celui ou de ceux qu'il choisissait, ainsi que de préserver le caractère secret du contenu de l'enseignement. Ce caractère était, semble-t-il, bien plus marqué pour les activités ayant trait au kanuragan et pour celles attenantes au principe d'autorité traditionnelle.

Les autres activités" étaient retransmises suivant un même schéma type. Ce système ancien est appelé nyantri, forme verbale de la base sanskrite santri, qui veut dire à la fois apprendre chez un maitre et résider chez lui. Notons que le terme santri signifiait aussi " élève pèlerin ", eu égard à la tradition de pérégrination courante à Java pour ceux qui "partent en quête de connaissances » (ngèlmu). Nyantri désignait aussi la relation corrolaire de la pratique de matrilocalité- qui lie l'époux à son beau-père, considérée comme une relation valorisée propice au transfert des savoirs et savoir-faire; dans les modes de représentation, l'épouse est d'ailleurs souvent associée à l'acquisition du savoir. Aujourd'hui, santri désigne les élèves des écoles musulmanes et a donné forme au terme affixé pesantren ( pe+santri+an), "école coranique", la tradition de pérégrination s'étant maintenue en contexte musulman.

En convergence avec ce cadre de transmission traditionnel, chez TT et chez ML, le maitre retransmet ses techniques à au moins un de ses enfants, état de fait encore très répandu. Chez TT Mbah Budi a retransmis son savoir-faire à son fils unique (il a par ailleurs trois filles). Chez ML, le fils aîné semblait destiné à reprendre le flambeau lorsqu'il s'est lancé comme son père dans la carrière politique (ses sœurs et beauxfrères sont aussi pratiquants). Dans ces deux cas-là et dans la plupart des autres, la succession du maître dépend néanmoins du niveau de maîtrise et d'expérience acquis. Il s'établit sur le mode du consensus entre les principaux responsables. Cela n'exclut pas les désaccords, les tensions, voire les fractionnements de l'organisation.

Concernant pratique et transmission, il faut ici insister sur la notion de ngèlmu. Tout d'abord, il s'agit de la forme affixée de èlmu qui signifie "savoir ", " connaissance », « science ». Les Javanais emploient presque systématiquement la forme verbale active ngèlmu, car pour eux, la dimension pratique et vécue est prépondérante. Quelqu'un qui maitrise une pratique, un savoir, une connaissance, une science, est quelqu'un qui agit avant tout pour se l'approprier. L'activité empirique et le vécu de l'action dominent nettement l'activité théorique et la conceptualisation.

En parallèle, si la connotation de recherche ésotérique impliquée par ngèlmu est la plus marquante, le terme s'applique tout aussi bien à l'acquisition des savoirs religieux et des sciences modernes, ce qui les situe à un niveau de valeur comparable à celui des savoir-faire traditionnels javanais les plus élaborés. Le système javanais est donc ouvert à l'introduction d'éléments extérieurs, mais il maintient la nécessité de les intégrer selon une perspective javanaise, notamment celle d'accompagner les nouvelles pratiques d'un laku, d'une concentration intérieure s'appuyant sur une ascèse plus ou 
moins poussée (chacun est libre d'en déterminer la teneur), en vue de favoriser le vécu du cheminement à suivre.

Réseaux et relations internes

70 Le maître de TT est intégré à un réseau relationnel multiple. Le noyau dur en est constitué par ceux qui veulent véritablement approfondir les connaissances de kanuragan et qui fréquentent régulièrement la maison du maître, même en dehors des phases propres d'apprentissage. Bon an mal an, ils sont environ une vingtaine. Il y a ensuite les anciens élèves de Mbah Budi qui ont approfondi son enseignement et dont certains continuent à participer aux activités pour garder le contact et soutenir les efforts du maître en vue de maintenir actifs les savoir-faire javanais. L'ensemble de ce sous-groupe approche la cinquantaine d'individus. Mbah Budi a des contacts dans différents villages de la région de Yogyakarta, ainsi que dans la petite ville isolée de Wonosari qui est réputée avoir accueilli des réfugiés hindo-bouddhistes à l'époque de l'expansion des royaumes musulmans dans la région (seconde moitié du XVI ${ }^{\mathrm{e}}$ siècle). $\mathrm{Au}$ travers de ces contacts, des groupes de jeunes gens viennent périodiquement se former chez lui aux techniques de kanuragan. Il reçoit ainsi, six à huit fois l'an, un groupe d'une vingtaine de personnes. Enfin, les commerçants sino-indonésiens évoqués en première partie l'emploient pour effectuer des cérémonies propitiatoires ou de conjuration du mauvais sort.

Outre ces réseaux, nous l'avons vu, le maître est aussi très consulté de façon individuelle pour toute sorte de problèmes: maladie, fugue, folie, recherche de l'âme sœur, départ sur le front guerrier, préparation des élections, etc. J'insiste sur ces différents points pour bien montrer la richesse que ce personnage a développée au niveau de l'expérience propédeutique et relationnelle. Ainsi l'attribution du jeûne aux élèves qu'il forme prend-elle un sens particulier si on la compare à la façon dont les mouvements respiratoires sont transmis chez ML.

Dans ce cas-là, le responsable local n'est pas le maître, mais l'entraîneur. L'expérience qu'il lui est permis de développer de façon quasi exclusive est celle qu'il a acquise au sein de l'école. De même, les techniques qu'il transmet sont exclusivement celles de l'école. Il apparaît ainsi que la transmission s'appauvrit par rapport à l'acquis initial du maître - dont le contenu et le donné relationnel sont bien plus larges que ce qu'il enseigne (à l'instar de Mbah Budi). Il apparaît de même que le partage qui s'opère entre les différents assistants et entraîneurs s'accompagne d'un aplatissement relationnel; on ne dit pas, par exemple, d'un entraîneur qu'il « fait descendre son savoir » (nurunaké èlmuné) comme on le dit d'un maître. Dans le même temps que savoir et savoir-faire sont transmis à un plus grand nombre, la qualité relationnelle perd, elle aussi, en densité. J'ai souligné par ailleurs les déclarations de hauts responsables d'écoles suivant lesquelles l'entraîneur manque fréquemment de maturité (Grave 2001: 240, 268-69, 305-306). Cela est dû au fait qu'il évolue dans un environnement comparativement protégé et formalisé. Les niveaux de pratique sont clairement définis et des épreuves d'examens sanctionnent le passage d'un niveau à l'autre. L'objectif à atteindre prend beaucoup plus d'importance que le rapport au vécu de l'action et de la relation d'apprentissage. Dès le départ, il suffit d'ailleurs au pratiquant de s'inscrire auprès d'un groupe d'entraînement -ouvert à tout public- sans avoir à passer par un réseau relationnel particulier. Ces observations sont à mettre en rapport avec l'affaiblissement rituel relevé chez ML.

Temporalité des activités 

javanais, lequel était originellement luni-solaire et suivait l'ère Saka. Lorsque le calendrier musulman a été adopté par le sultan Agung en l'an 1633 de notre ère, ce dernier l'a fait partir de l'année Saka 1555 et non de l'année musulmane du moment, 1043, pour maintenir la spécificité javanaise vis-à-vis de l'islam. Néanmoins, le comput javanais ${ }^{12}$ qui s'élabore selon des cycles de 5 et 7 jours, est un des points fondamentaux qui participent de la continuité rituelle des activités de l'école TT avec l'ensemble du système cérémoniel javanais. toutefois pas empiéter sur la plage horaire de l'une des cinq prières quotidiennes (en général la "prière du soir ", salat magrib). Ces remarques valent en partie pour les autres activités, sauf pour les cérémonials de passage de niveau qui sont préférablement tenus après la prière musulmane centrale du vendredi. La réunion nationale annuelle des centres de l'école est diversement tenue soit en coïncidence avec une fête musulmane, soit avec l'anniversaire du maître. Le pèlerinage sur la tombe du sultan Agung coïncide, quant à lui, avec la date de naissance de ce roi.

Pour ML, il y a donc interpénétration du temps national et du temps javano-musulman. Le comput javanais évoqué ci-dessus n'intervient pas de façon formelle, sauf dans des centres autonomes de ML où les maîtres inscrivent explicitement leur pratique dans le kejawèn (Grave 2001 : 151-153).

Le rapport aux valeurs sociales

Les pratiques de l'une et l'autre écoles se développent donc suivant l'acquisition progressive de capacités similaires: maîtrise physique et émotionnelle, santé, invulnérabilité, pratiques thérapeutiques et propitiatoires, accroissement de l'autorité spirituelle, etc. Nous avons néanmoins pu constater que les techniques en cause varient d'un cas à l'autre.

Dans le cas de TT, il apparaît que la pratique s'adresse à deux catégories de personnes englobant les différents réseaux et cas de figure évoqués plus haut : celles qui sont plus ou moins intégralement impliquées dans le mode relationnel «javaniste » (kejawèn) et celles qui n'y sont impliquées que partiellement, eu égard à leurs autres activités ou préoccupations (religieuses, professionnelles...). Néanmoins, dans les deux cas, elles se sentent interpellées par ce que représente le kejawèn. Et cela, d'autant plus que la formation octroyée s'adresse à des personnes -comparativement à ML- globalement plus mûres en âge et en matière de sensibilisation à leur appartenance socioculturelle. Les membres concernés sont donc nettement moins importants numériquement chez TT que chez ML, mais ils sont socialement plus homogènes.

Chez ML, on recrute plus en amont puisque les membres sont globalement plus jeunes. Les techniques de base s'adressent à un public séculier élargi, qui recouvre néanmoins plusieurs appartenances religieuses. Ce n'est qu'en progressant dans la pratique que l'adhésion à l'islam devient sinon obligatoire, du moins nécessaire à partir d'un certain point. En deçà, les idéaux républicains énoncés par le premier président d'Indonésie, Soekarno, se présentent comme des valeurs de référence. Voici l'idée explicitement développée par Bapak Effendi : les techniques de tenaga dalam sont universellement praticables et l'école Marga Luyu, en réunissant des groupes socioculturels et des confessions issus de différents horizons, travaille efficacement à lutter contre les tensions interethniques et interreligieuses. 
79 Du point de vue national, et nationaliste, seules les techniques respiratoires et les mouvements corporels sont suffisamment neutres pour être acceptés par un très large éventail de population, comme c'est le cas avec ML. L'introduction d'éléments musulmans dans la suite de la pratique dresse des limites : ceux qui ont épousé une autre confession religieuse et ne veulent en aucun cas se partager ne viendront pas, ou alors -s'ils ont commencé en niveau de base- ils partiront.

80 Certaines écoles de tenaga dalam ont ainsi choisi de dispenser un enseignement et de former à des techniques exemptes de connotations religieuses, favorisant de la sorte une laïcisation de la pratique. C'est par exemple le cas de Satria Nusantara, «Les chevaliers de l'Archipel», ou de Merpati Putih, «La colombe» (concernant cette dernière, cf. Grave 2001 : 209-312), qui font partie des écoles ayant formé depuis leur création plusieurs centaines de milliers de personnes. Ces deux écoles sont, pour beaucoup, imbriquées dans un système de valeurs nationales séculières. Les techniques sont rationalisées dans le sens où une collaboration très poussée s'instaure avec les milieux médicaux et sportifs. Cela implique une formalisation des systèmes de transmission encore plus poussée que chez ML. Le support écrit est utilisé de façon quasi systématique, les entraîneurs ont en leur possession l'intégralité du cursus consigné en un ou plusieurs fascicules. Chez Satria Nusantara, les élèves qui veulent monter en niveau quatre doivent même écrire un mémoire concernant les techniques de l'école et les connaissances bio-médicales. L'école est d'ailleurs implantée dans un hôpital expérimental de Surabaya (Java Est), où ses membres habilités soignent des personnes atteintes de différentes maladies (rhumatismes, hypotension, diabète, maladies respiratoires, maladies cardiaques...), en leur enseignant techniques respiratoires et mouvements de façon adaptée et, dans les cas les plus graves, en utilisant leur propre tenaga dalam par transfert au niveau des mains.

81 Il faut néanmoins souligner le fait que même de telles organisations conservent un ancrage marqué dans leur fondement socioculturel. Cet ancrage concerne plus particulièrement les membres avancés ou très avancés. Ayant acquis un certain recul et une autonomie relative par rapport aux techniques de l'école, ils consultent volontiers d'autres maîtres -y compris des « sages » (sesepuh) tels Mbah Budi- et renouent avec les techniques ancestrales d'ascèse (bains nocturnes dans les rivières, jeûnes, méditation sur les lieux sacrés...). S'élabore ainsi une reviviscence de ces pratiques, sur la base de systèmes de transmission urbanisés, mais généralement encore en relation avec les réseaux traditionnels.

82 Si les techniques ont été pour beaucoup épurées de leurs composantes rituelles locales et rendues adaptables à des contextes sociaux indifférenciés, la marque locale reste aussi présente au travers de l'histoire de l'école, de sa région, de son ou ses fondateurs et de la folklorisation d'éléments rituels.

83 Cela signifie que l'on a -à l'instar de chez ML- un premier niveau où la profondeur rituelle et religieuse est pour beaucoup désactivée, et un second où elle est maintenue, quoique se modifiant au contact des systèmes de valeurs et de transmission à tendance universaliste. Le rapport à la localité se modifie du fait de l'intégration de la pratique aux modèles de référence nationaux. Mais dans le même temps, ce même rapport se pose lui-même comme une référence -en l'occurrence javanaise- auprès des autres contextes sociaux qu'il pénètre. Il s'avère d'ailleurs que les Javanais exportent beaucoup leurs techniques de pencak, de tenaga dalam et même de "mystique " (kebatinan) vers les autres régions d'Indonésie, et à l'étranger. Il arrive de plus, dans le 
cas d'écoles comme Marga Luyu, que des Occidentaux, au départ complètement athées, en viennent, par effet-retour, à s'intéresser à l'islam, voire à se convertir. Dans de tels exemples, la pénétration idéologique suit celle des pratiques : les techniques suscitent l'intérêt, lequel en vient à s'étendre au système de valeurs. Dans ce cas, un contexte social sécularisé sert d'appui au système religieux. Il sert de passerelle et oriente la possibilité des choix.

Revenons à TT. Ici le problème se pose de façon différente, dans la mesure où l'appartenance religieuse quelle qu'elle soit -voire l'absence d'appartenance-, ne représente pas une entrave à la pratique. Mbah Budi encourage même ses élèves à participer activement aux activités religieuses de leur confession d'appartenance. Cela signifie qu'il ne ressent pas ces activités - du fait de leur formalisme poussé- comme entrant en concurrence avec la sienne. C'est aussi une façon de se prémunir des attaques des groupes religieux orthodoxes à son égard. Cela signifie encore que son système de valeurs est proche du religieux (religieux entendu ici comme registre d'activité autonome du registre rituel), mais plus éloigné que ne l'est ce dernier des références séculières. L'État-nation indonésien, dans sa constitution originelle, ne laisse pas de place aux types de cultes non formalisés. Pourtant, dans la pratique, une certaine tolérance domine. Il reste que cette tolérance n'est pas partagée de façon égale selon les régions et selon l'origine sociale.

Pour Mbah Budi, il est difficile de naviguer entre deux eaux comme le font les écoles de pencak ou de tenaga dalam. Pas de collaboration possible avec les milieux médicaux ou sportifs et, surtout, pas de possibilité d'introduire les modes de transmission modernes, à moins de folkloriser la pratique. Il ne lui reste qu'à ignorer les pressions qu'exercent sur son groupe les partisans convaincus de la sécularisation et de l'orthodoxie religieuse, et à susciter le soutien de ceux qui reconnaissent quelque valeur au mode de pratiques et de relations qu'il défend.

La transmission : entre dimension technique du social et dimension sociale du technique

Cette recherche, on l'aura noté, accorde une place particulière à la transmission. L'approche que je développe s'intéresse de près aux techniques; néanmoins, il me semble bien qu'il y a tout intérêt à comprendre ces dernières dans le cadre élargi de la transmission et de l'apprentissage plutôt que l'inverse. François Sigaut (1991: 33) a bien posé le problème en constatant que ces deux paradigmes sont généralement étudiés comme « deux rubriques séparées, sans connexions entre elles ». Pourtant, s'il est possible d'étudier des techniques sans étudier le procès de leur pérennisation, il est plus difficile de parler transmission sans éléments techniques.

La comparaison que j'ai établie dans les pages qui précèdent concorde avec cette idée que les techniques changent (en partie au moins), mais que la transmission -au même titre que les hommes- demeure : elle englobe les premières. Si elle change, cela est moins visible, moins flagrant que pour les techniques: le changement technique observé pousse facilement à surestimer le changement relationnel et le changement de valeurs impliqués. Les exemples des écoles Marga Luyu, Merpati Putih et Satria Nusantara indiquent qu'en parallèle à l'introduction de valeurs religieuses formelles, nationalistes et/ou scientifiques, les éléments javanistes se réifient aux niveaux de base mais se maintiennent aux niveaux élevés de pratique.

En définitive, on ne peut pas dire que ce sont les nouveaux systèmes de valeurs qui influencent le javanisme plutôt que le contraire. D'un point de vue interne, le 
javanisme subordonne les autres systèmes de valeurs et se développe. Si l'on en reste à un point de vue externe, on peut effectivement parler de changements conséquents, mais ce serait surinterprétatif par rapport à la relation à l'altérité des Javanais. Comme l'a souligné Marshall Sahlins (1999), il convient d'observer la capacité d'interprétation et d'adaptation des sociétés avant de les qualifier d'acculturées. S'appuyer sur les processus de transmission comme interface des techniques et des valeurs nous aide à y voir plus clair sur ce point.

Il faut bien sûr recueillir des pratiques dont on sait qu'elles sont par essence menacées". Mais il faut aussi -et surtout- s'intéresser aux modes relationnels de perpétuation qu'elles impliquent. Cela peut aller loin, car dans l'absolu, il convient de prendre en compte les éléments sociaux généraux et formateurs qui contiennent en germe la technique que l'on étudie. J'ai présenté ici, pour le contexte social javanais, quelquesuns de ces éléments (système nyantri, matrilocalité, classifications temps/espace/cycle de vie, pérégrination, facultés humaines). Pour bien saisir l'ancrage d'une pratique, il convient d'être aussi exhaustif que possible.

Nous arrivons ainsi à un résultat qui se rapproche de la description monographique. Ce type d'approche a été prôné par Christian Pelras (1973) et Jean-Pierre Digard (1979: 81-83) comme base nécessaire de la formation d'une théorie générale non pas des seules techniques, mais «de la détermination réciproque des systèmes techniques, économiques, sociaux, etc.» (Digard 1979: 83). Dans l'optique que je propose, il ne s'agit pas non plus d'une description monographique de type classique qui s'intéresserait, pour beaucoup, aux éléments endogènes du groupe étudié et qui suppose une homogénéité sociale marquée, laquelle n'est plus vraiment de mise de nos jours. Il s'agit de comparaisons internes, similaires à celles que je mets en œuvre dans cet article, qui permettent à la fois de placer en perspective comparative les différentes techniques d'une même catégorie et d'évaluer la part d'innovation, ou plutôt d'altérité -terme moins ethnocentré- qui les concerne.

91 Encore une fois, dans cette vue que je défends, ce sont les systèmes de transmission qui englobent le technique. Ils l'englobent du fait que ce sont eux qui garantissent le succès ou le dépérissement de telle ou telle technique parce qu'ils sont partie intégrante du système de relations et de valeurs sociales, a fortiori dans des contextes où la valeur n'est pas séparée du fait (Dumont $1983: 248 / 9$ ) et où la dichotomie " théorie-pratique » ne fait pas sens.

92 Nous touchons là le cœur du problème qui oppose, en partie au moins, les technologues aux symbolistes depuis la prise de position d'André Leroi-Gourhan en faveur de l'approche technologique (cf. notamment Bulletin de techniques \& culture $\mathrm{n}^{\circ} 1,1976$; Digard 1979; Bonte 1986; Cresswell 2002 ; Guille-Escuret 2002; Sigaut 2002), problème qui tourne autour de la question de la situation des techniques dans la configuration sociale globale et de la délimitation, dans le sillage d'André-Georges Haudricourt, de l'objet de la «technologie science humaine ». La question est vaste et je ne peux ici qu'évoquer rapidement quelques points afin de mieux préciser ma perspective.

93 S'il est évident que le technique est partie intégrante du social, il est aussi évident que les techniques sont, dans les faits, partout. Aliette Geistdoerfer (1977 : 34) reconnaît que la technique n'est pas du seul ressort de la technologie matérielle dans son rapport à la matière, mais il faut bien constater que les études françaises de technologie, qu'elles soient descriptives ou théoriques, traitent essentiellement de l'environnement matériel 
et du rapport à la matière, point qui a été souligné et critiqué par Pierre Bonte (1986 : 38-39).

Comme cet auteur le pointe justement : « La distinction entre activité rituelle et activité technique n'est pas pertinente dans toutes les sociétés » $(1986: 47)$. Jean-Pierre Warnier (1999 : 28-30) -qui axe sa démarche méthodologique sur un rapport très marqué à la culture matérielle- souligne d'ailleurs l'intérêt poussé de Marcel Mauss pour la dimension technique des actes rituels et religieux dans ses textes d'avant 1920. Dans Les techniques $d u$ corps, Mauss lui-même conclut sur ce que certaines pratiques corporelles et respiratoires (il s'agit des techniques yogiques d'Inde et taoïstes de Chine) sont susceptibles de nous apprendre dans la perspective de l'« homme total $»^{14}$ qu'il est en train d'ébaucher; il nous faut bien constater que ces techniques ne sont enclavées ni dans la matière ni dans la matérialité ${ }^{15}$. C'est dans cette perspective de non-rapport systématique au matériel que peut se comprendre le fait technique de façon élargie.

Partant, le fait technique de base est celui de l'acte de transmission. Il permet d'accéder aux échanges véritables qui s'opèrent entre les techniques et les valeurs. Que l'approche technologique ne se suffise pas à elle-même semble être un point généralement acquis. Pour Pierre Bonte (1986: 47), par exemple :

«La relecture technologique des faits techniques ne suffit pas, elle peut même, au nom d'une représentation rationalisante des techniques, masquer l'essentiel: l'inscription du fait technique, fait social, fait culturel, dans une société, dans une culture donnée."

Il poursuit un peu plus loin :

«L'anthropologie des techniques pourrait se constituer lorsque [...] on poserait que les valeurs sociales sont la finalité de l'activité technique et la condition de sa mise en œuvre. » $(1986: 47)$.

Par rapport à ce type d'assertion, nous devons dorénavant dépasser le simple saut du technique au social ou vice-versa. S'il me semble acquis que la transmission englobe le technique, je n'affirmerais pas que les valeurs sociales englobent l'un et l'autre de façon univoque. Entre, d'une part, les perspectives symbolistes, représentationnelles ou structurales qui subordonnent nettement, et le technique et les systèmes de transmission qui le concernent, et d'autre part les perspectives technologiques qui valorisent le technique et revendiquent pour lui le statut de fait social total, il ne me semble pas nécessaire de trancher à tout prix.

98 Je tendrais plutôt à dire qu'il s'établit une relation d'inter-influence entre le technique et les valeurs sociales. De même que les techniques se reformulent à chaque génération et à chaque instant, les valeurs et les structures sociales ne revêtent pas la stabilité qu'on leur prête bien souvent (cf. la critique de l'analyse structurale lévi-straussienne du mythe par Jack Goody 1994 : 182).

Quant au contexte javanais sur lequel je travaille depuis de nombreuses années, il est clair que des techniques comme celles que je décris dans cet article ont servi d'appui et d'ancrage à l'introduction de l'islam à Java. Il est certain que si l'islam ne disposait pas de techniques de jeûne, de formules visant à acquérir différentes sortes de protection et différentes capacités, de techniques respiratoires (soufisme), etc., il n'aurait pu attirer l'attention des Javanais.

100 Ceci n'empêche pas l'élaboration parallèle d'un processus formel d'instauration des valeurs de l'islam, mais, selon la métaphore consacrée, il est aux techniques un peu ce que la poule est à l'œuf. À moins de disposer en conséquence d'une montagne de 
données historiques et ethnographiques factuelles que nous n'avons pas, il est impossible de trancher : préséance des valeurs sur les techniques ou des techniques sur les valeurs. Bien sûr, on peut toujours dire que même si l'adoption de techniques a précédé celles de valeurs formelles -comme il semble que ce soit le cas pour Java concernant l'islam (voir notamment Kamajaya 1985 et Zoetmulder 1990)-, c'est l'adoption des valeurs qui prime dans l'absolu. Ma réponse sera en trois temps.

Tout d'abord, la mise en place de valeurs sociales est très longue et il s'agit d'un processus continu; les valeurs de demain sont déjà potentiellement présentes aux travers de choix qui s'opèrent aujourd'hui au niveau pratique, les relations de parenté et d'affinité par exemple. Placer la valeur a priori comme englobante revient en gros à énoncer un résultat, à subordonner les moyens aux fins. Par prudence, il faudrait l'énoncer comme contextuellement englobante, ou englobante relativement à tel et tel éléments. Dans cet ordre d'idées, la constatation de Catherine Choron-Baix (2000b : 359) d'une somme variable d'éléments transmis «involontairement " lors du "procès de transmission " la conduit à nous mettre en garde d'interpréter ce processus comme étant exclusivement linéaire. Maurice Bloch (1991) a d'ailleurs bien souligné la part d'inconnu que revêt tout mode de transmission, même ceux dont on pense qu'ils sont très formalisés.

102 L'étude des systèmes de transmission nous place ainsi au plus près du contextuel et du relatif. Elle permet de développer une analyse plus détaillée qui tient compte à la fois de la dimension technique du social et de la dimension sociale du technique. Elle permet par exemple de différencier les modes plus traditionnels - qui valorisent davantage le vécu relationnel et le cheminement progressif comme cadre de l'acquisition des savoirs et savoir-faire- des modes de transmission formels influencés par l'orthodoxie religieuse, le sécularisme et plus largement par l'écrit comme support référentiel $\mathrm{du}$ centralisme politique- lesquels privilégient comparativement l'objectif à atteindre. Dans un cas, la technique est dans le relationnel, dans l'autre elle se sépare du relationnel.

Cette opposition n'est pas figée, un aller-retour continu s'établit de l'un à l'autre de ses pôles. On trouve dans ce texte des exemples de modes traditionnels chez Trah Tedjokusuman et dans le système nyantri, et des exemples de modes plus formels dans les écoles modernes de tenaga dalam. Pour ces dernières, on observe que l'introduction des valeurs religieuses orthodoxes et des valeurs séculières s'accompagne effectivement d'une moralisation du comportement (la séparation du fait et de la valeur), qui est compensée par la pratique quantitativement très développée du tenaga dalam ressentie comme concrète, tant au niveau de l'entraînement des facultés perceptives et émotionnelles que du système relationnel qu'elle implique'.

Il reste que le mouvement-retour de compensation peut se traduire par la formation de pôles radicaux. On a notamment pu l'observer en Indonésie lors de l'attentat de Bali du 12 octobre 2002 perpétré par des musulmans Javanais radicaux. L'étude des modes de transmission, en ce qu'elle permet de conjuguer ce qui est de l'ordre des savoir-faire et de l'ordre des valeurs, doit aussi permettre de traiter de sujets aussi importants que celui-ci. 


\section{BIBLIOGRAPHIE}

Références

Bloch, Maurice

1991 « Language, Anthropology and Cognitive Science », Man 2 : 183-198 (Repris dans How we think they think - Anthropological Approaches to Cognition, Memory, and Literacy, 1998, Oxford Westview).

Bonte, Pierre

1986 « Les recherches ethnologiques sur les techniques en France après A. Leroi-Ghouran ", La Pensée 254 : 37-47.

Chevallier, Denis

1989 « Le programme savoir-faire et techniques », Terrain $12: 138-141$.

Chevallier, Denis \& Isac Chiva,

1991 «Introduction - L'introuvable objet de la transmission », in Savoir faire et pouvoir transmettre - Transmission et apprentissage des savoir-faire et des techniques. Paris : Éditions de la Maison des sciences de l'homme («Cahiers d'ethnologie de la France » 6) : 1-11.

Choron-Baix, Catherine (ed.)

2000a Ethnologie Française 83 : « Envers et revers de la transmission ».

Choron-Baix, Catherine

$2000 \mathrm{~b}$ « Transmettre et perpétuer aujourd'hui », Ethnologie Française 83 : 357-360.

Cresswell, Robert

2002 " Geste technique, fait social total - Le technique est-il dans le social ou face à lui ?», Techniques \& culture $40: 125-151$

Damais, Louis-Charles

1967 « Le calendrier de l'ancienne Java », Journal Asiatique : 133-141.

Digard, Jean-Pierre

1979 « La technologie en anthropologie : fin de parcours ou nouveau souffle? », L'Homme XIX-1 : 73-104.

Dumont, Louis

1983 Essais sur l'individualisme. Une perspective anthropologique sur l'idéologie moderne. Paris : Éditions du Seuil.

Friedberg, Claudine

1985 « La santé à Bali - Le plein, le tempéré et le juste milieu », L'ethnographie 96-97 (2).

Geertz, Clifford

1960 The Religion of Java. Chicago : The University of Chicago Press.

Geistdoerfer, Aliette 
1977 « Description des techniques matérielles », Les Cahiers du Centre d'études et de recherches marxistes $132: 33-52$.

Goody, Jack

Entre l'oralité et l'écriture. Paris : Presses Universitaires de France.

Grave, Jean-Marc (de)

1996a « Une école de pencak silat prise sur le vif », Archipel $52:$ 77-93.

$1996 \mathrm{~b}$ « Une école catholique de pencak silat - Tunggal Hati Seminari », Archipel 52 : 65-75.

2001a Initiation rituelle et arts martiaux - Trois écoles de kanuragan javanais. Paris : Archipel-

L'Harmattan.

$2001 \mathrm{~b}$ « Hubungan pendidikan formal dan non-formal di Indonesia - Pencak silat, tari dan citacita para pendiri pendidikan di Indonesia » (Relation entre éducation formelle et non formelle en Indonésie du point de vue des fondateurs de l'éducation indonésienne), Suluah I-2 : 7-10.

2003 « Dossier sur les arts martiaux d'Asie du Sud-Est (I) », La Lettre de l'AFRASE 61 : 15-19.

2007 «Quand ressentir c'est toucher - Techniques javanaises d'apprentissage sensoriel », Terrain 49 : 77-88.

Guille-Escuret, Georges

2002 « Efficacité technique, efficacité sociale. Le technique est-il dans le social ou face à lui ? », Techniques \& culture $40: 1-34$.

Jordaan, Roy E.

1985 Folk Medicine in Madura (Indonesia). Thèse de doctorat, Leiden : Rijksuniversiteit.

Kamajaya

1985 Serat Centhini Latin I-XII. Yogyakarta : Yayasan Centhini Yogyakarta.

Mauss, Marcel

1999 « Les techniques du corps » in Sociologie et anthropologie, Paris : Presses Universitaires de France (1ère édition, « Les techniques du corps », Journal de Psychologie $32(3-4), 1936)$ : 363-386.

Pelras, Christian

1973 « Technologie, ethnographie, ethnologie » in L'Homme hier et aujourd'hui : recueil d'études en hommage à André Leroi-Gourhan. Paris : Éditions Cujas : 715-730.

Pemberton, John

1994 On the Subject of Java. Ithaca : Cornell University Press.

Sahlins, Marshall

1999 Les Lumières en anthropologie? Nanterre : Société d'Ethnologie.

Sigaut, François

1991 «L'apprentissage vu par les ethnologues - Un stéréotype ? » in Savoir faire et pouvoir transmettre - Transmission et apprentissage des savoir-faire et des techniques. Paris : Éditions de la Maison des sciences de l'homme («Cahiers d'ethnologie de la France » 6) :34-42.

2002 « La formule de Mauss », Techniques \& culture $40: 153-168$.

Techniques \& culture 1 
1983 Paris, Éditions de la Maison des sciences de l'homme.

Warnier, Jean-Pierre

1999 Construire la culture matérielle - L'homme qui pensait avec ses doigts. Paris : Presses

Universitaires de France.

Woodward, Mark R.

1989 Islam in Java - Normative Piety and Mysticism in the Sultanate of Yogyakarta. Tucson : The University of Arizona Press.

Zoetmulder, Pietr J.

1990 Manunggaling Kawula Gusti, Pantheïsme dan monisme dalam sastra suluk Jawa. Jakarta : Gramedia.

\section{NOTES}

1. L'enseignement étant octroyé en javanais, les termes vernaculaires présentés ici sont javanais.

2. Le terme mantra, d'origine sanskrite, désigne une formule d'invocation ou de protection.

3. Mbah est un appellatif de respect tiré du terme de parenté embah " grand-parent ». Il désigne les personnages charismatiques d'un lieu donné, qui ont acquis une riche expérience et de nombreuses connaissances.

4. Comprenant notamment les fleurs sritaman (des termes sri, « lumineux, éclatant de beauté » et taman, « jardin ») : rose (mawar), cananga (kenanga), jasmin (mlathi), champac (kanthil).

5. Il s'agit du croisement de la semaine de sept jours et de la " semaine de marché » (pasaran) de cinq jours (legi, paing, pon, wagé, kliwon). Sur le calendrier javanais, cf. Grave 2001 : 343-344 et Damais 1967.

6. Des tombes, des plages, des grottes, des rochers, des sources, des rivières, des montagnes...

7. Le PDI (Partai Demokrat Indonesia) et le Golkar (Golongan Karya), notamment.

8. L'enseignement étant pourvu ici en indonésien (langue nationale), les termes concernant cette école sont indonésiens.

9. L'affiliation, bien que non authentifiée, révèle comme pour TT le caractère hautement valorisé des pratiques en cause.

10. Pour d'autres mises en pratique que les soins en tenaga dalam, celles ayant trait notamment au développement de la perception tactile et des capacités proprioceptives, voir Grave 2007. J'y effectue une mise en perspective avec d'une part la perception en Europe au XVIII ${ }^{e}$ siècle, et d'autre part les connaissances neurophysiologiques et psychophysiologiques actuelles traitant de l'action et de la perception.

11. Théâtre et danse classiques, théâtre d'ombre, art martial pencak, littérature, forge des kriss ou des instruments en métal du gamelan, confection des figurines en cuir du théâtre d'ombre, orfèvrerie, etc.

12. Damais (1967) a montré que le cycle de 5 jours était certainement proprement nousantarien.

13. C'est le programme que s'était fixé la Mission du patrimoine ethnologique (Chevallier 1989). Ce programme a d'ailleurs suscité un début d'effort de réflexion sur 
la transmission (Chevallier \& Chiva 1991), mais ce type d'initiative reste très ponctuel (voir aussi Choron-Baix 2000).

14. C'est-à-dire l'homme dans ses dimensions physiologique, sociale et psychique.

15. Notons aussi que quatre et trois paragraphes plus haut (1993 : 385), il insiste sur le rôle de l'éducation par le corps, laquelle nous ramène aux questions de transmission. 16. Clifford Geertz (1960 : 158) notait déjà que le pentjak qu'il avait observé dans les années 1950 dans les écoles coraniques compensait avantageusement la dimension « austère » de l'islam.

\section{RÉSUMÉS}

L'initiation rituelle javanaise présente un double aspect : transmission traditionnelle des techniques sous le sceau du secret du point de vue interne, et, du point de vue externe, méconnaissance du sujet par les nombreuses générations de chercheurs qui ont travaillé sur Java. Pour combler cette lacune, le présent article décrit deux écoles -l'une javaniste, l'autre musulmane- au-travers du mode de perpétuation des techniques utilisées et en référence étroite aux systèmes de valeurs respectifs à chaque groupe. La comparaison qui s'ensuit révèle une opposition assez importante entre le javanisme et l'islam avec le maintien, toutefois, d'un schéma global javanais. En fin de texte, l'auteur s'appuie sur les réflexions de différents anthropologues pour souligner la nécessité de revaloriser le thème de la transmission -interface privilégiée entre le technique et le social- comme paradigme de recherche en anthropologie. Le contexte étudié ici, notamment confronté à la résurgence de l'islam radical javanais, pose la question de base du rapport à l'action : qu'elles sont les implications sociales de la distanciation poussée visà-vis de la dimension pratique? Cette distanciation se prolonge-t-elle dans la dimension relationnelle? Quel rôle jouent les idéologies universalistes dans ce processus?

\section{INDEX}

Mots-clés : initiation, javanisme, islam, transmission, système de valeurs. 